\title{
01AP03-5 The effectiveness of prophylactic doses of intravenous nitroglycerin in preventing the incidence of myocardial ischaemia under general anaesthesia; A systematic review and meta-analysis
}

\section{Hiroshi Hoshijima ${ }^{1}$, DDS, PhD,}

Yohei Denawa ${ }^{2}$, MD, Risa Takeuchi ${ }^{1}$, DDS, PhD, Takahiro Mihara ${ }^{3}, \mathrm{MD}, \mathrm{PhD}$, Zen'ichiro Wajima4 ${ }^{4,5}, \mathrm{MD}, \mathrm{PhD}$, Hiroshi Nagasaka ${ }^{1}, \mathrm{MD}$, PhD

1 Department of Anaesthesiology, Saitama Medical University Hospital, Saitama, Japan

2 Department of Internal Medicine, Drexel University College of Medicine, PA, USA

3 Departments of Anaesthesiology and Critical Care Medicine, Yokohama City University Graduate School of Medicine, Kanagawa, Japan

4 Department of Anaesthesiology, International University of Health and Welfare Shioya Hospital, Tochigi, Japan

5 Department of Anaesthesiology, International University of Health and Welfare Hospital, Tochigi, Japan

\section{Background and Goal of Study:}

Nitroglycerin (TNG) has been shown to reverse myocardial ischaemia (MI). Observational studies have suggested that prophylactic doses of intravenous TNG is effective in reducing the incidence of intraoperative MI. However, the results of previous randomised controlled trials are not convincing. As a result, the effectiveness of prophylactic TNG in the prevention of intraoperative MI remains controversial. The purpose of this study was to evaluate whether prophylactic intravenous TNG can prevent the incidence of intraoperative MI. In addition, we also analysed haemodynamic changes (heart rate $[\mathrm{HR}]$, mean blood pressure $[\mathrm{MBP}]$, and pulmonary capillary wedge pressure [PCWP]) from in both pre and post anaesthesia induction as an indicator of the decrease in preload.

\section{Materials and Methods:}

This quantitative systematic review was performed according to the criteria outlined in the PRISMA statement. A comprehensive literature search was conducted to identify clinical trials which involved the administration of TNG in comparison to a placebo. The data from the individual trials were combined and the DerSimonian and Laird random-effects model was used to calculate either the pooled Relative Risk (RR) or the demonstrated weighted mean difference (WMD) in addition to the corresponding 95\% confidence intervals (CI). The data was analyzsed to clarify differences in the incidence of MI. We also analyzsed haemodynamic changes (HR, MBP, and PWCP) between TNG and the placebo. The heterogeneity of the results was examined by Cochrane's $\mathrm{Q}$ and $\mathrm{I}^{2}$ test.

\section{Results:}

\section{The result of selective data}

Using electronic databases, we initially identified 174 articles for review. Of these, 138 studies were excluded because they were unrelated studies. The other 36 articles were thoroughly checked to meet our inclusion criteria. Of these, 28 studies were excluded because they involved different drugs $(\mathrm{n}=$ $20)$ or included results that were unavailable $(n=10)$. Ultimately, 8 studies were identified by the defined search strategy, fulfilled the inclusion criteria and contained the necessary data for the planned comparison. The process of identifying eligible studies is illustrated in Figure 1, and the details of selected trials are summarized in Table 1.

\section{The result of analyses:}

Using electronic databases, we selected 8 trials for our review. Prophylactic intravenous $\mathrm{TNG}$ did not result in a decreased incidence of $\mathrm{MI}(\mathrm{RR}=0.61$; $95 \%$ CI, 0.33 to $1.13 ; p=0 . .12$; I2=55, Cochran's $Q=13.3$ ) (Fig 2). In terms of haemodynamic changes pre and post anaesthesia induction, intravenous TNG significantly reduced the MBP in comparison to the placebo. MBP; pre induction; WMD $=-7.27 ; 95 \%$ CI -14.2 to $-0.33 ; \mathrm{P}=0.04 ; \mathrm{I}^{2}=97 \%$; Cochrane's $\mathrm{Q}=136.9$, post induction; WMD $=-0.60 ; 95 \% \mathrm{CI}-10.3$ to $-2.09 ; \mathrm{P}=0.003 ; \mathrm{I}^{2}=68 \%$; Cochrane's $\mathrm{Q}=9.48$ ) (Fig 3, 4). In terms of haemodynamic changes (HR and PCWP), the effects of TNG were also similar to those of placebo during both before and after anaesthesia induction.
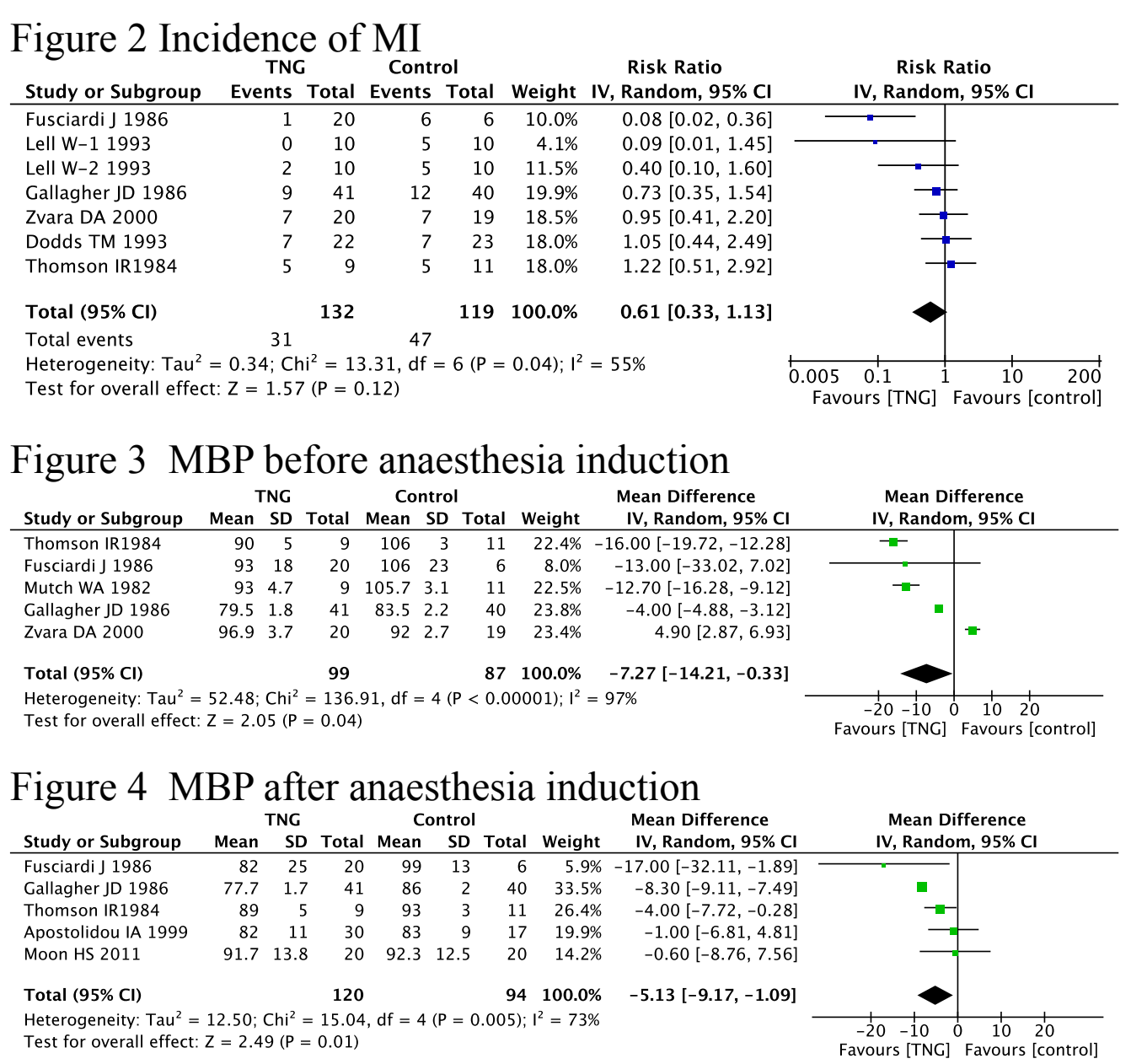

\section{Conclusions:}

Our analysis showed that prophylactic intravenous TNG does not reduce the incidence of intraoperative MI. Prophylactic doses of intravenous TNG significantly reduced the MBP both pre and post anaesthesia induction. From these results, prophylactic intravenous TNG provided little clinical benefit as it was not effective preventing the incidence of intraoperative MI.

\section{Figure 1 Flow chart}

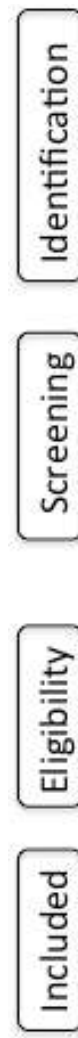

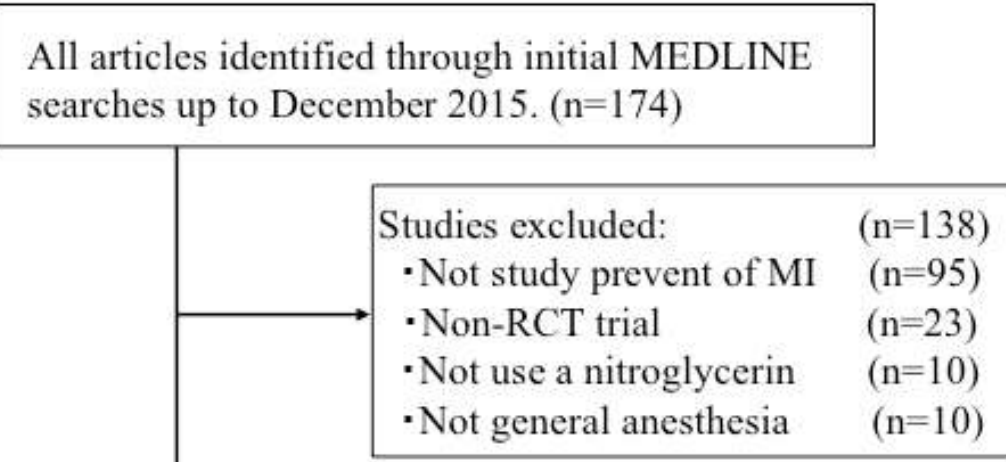

Studies which passed the initial screening $(n=36)$ - Difference drugs $\quad(\mathrm{n}=20)$ - Unavailable result $(\mathrm{n}=10)$

Studies included in the meta-analysis $(\mathrm{n}=8)$

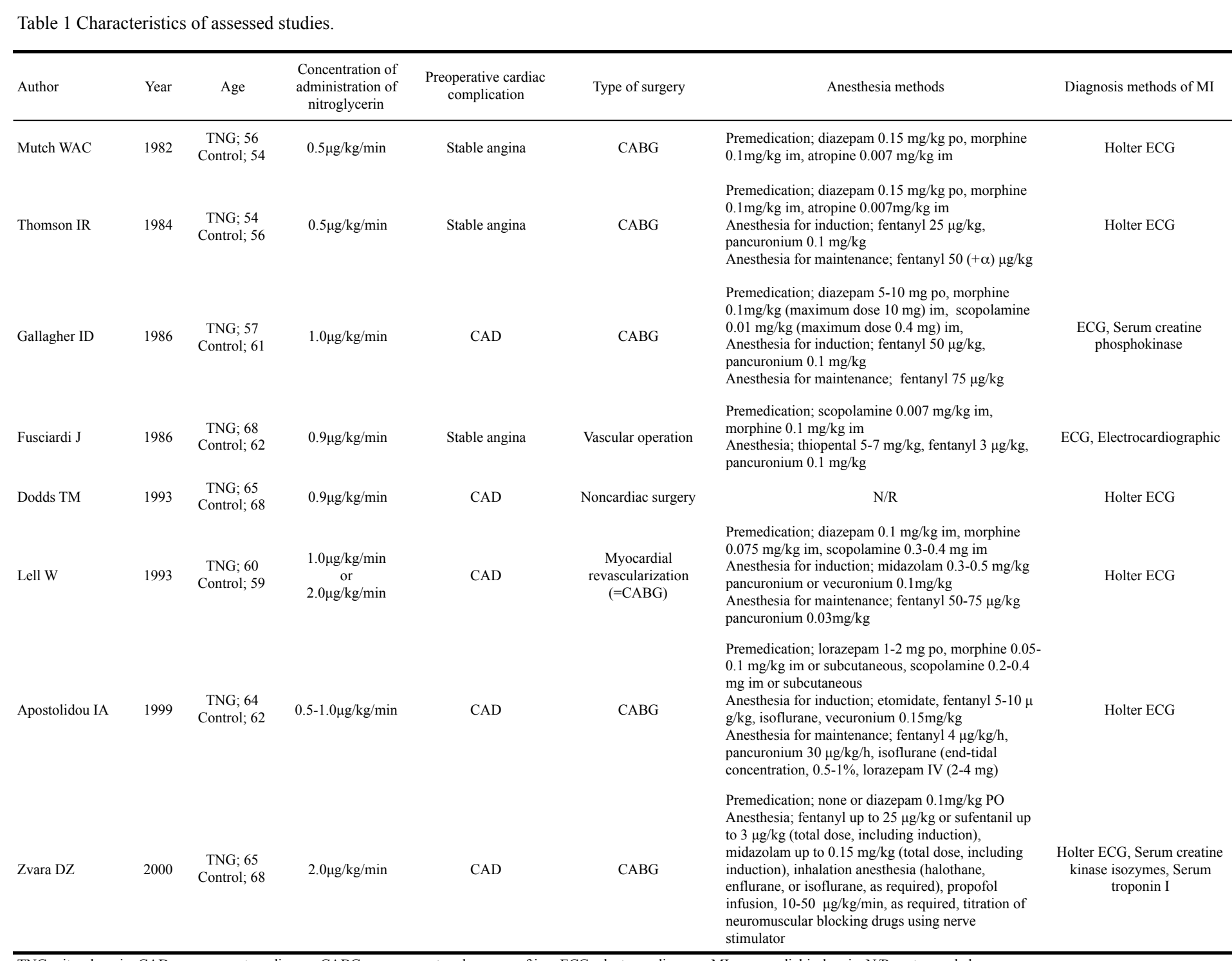

